\title{
Investigation of DRIE etching performance on signal quality of a SOI based pressure sensors for harsh environments
}

\author{
Piotr Mackowiak ${ }^{1}$, Fabian Meinecke ${ }^{1}$, Biswajit Mukhopadhyay², Thanh Hai Hoang², Quoc-Cuong. Dao², \\ Oswin Ehrmann ${ }^{1,2}$, Klaus-Dieter Lang ${ }^{1,2}$ and Ha-Duong Ngo $^{2,3}$ \\ ${ }^{1}$ Research Center of Microperipheric Technologies, Technical University Berlin \\ ${ }^{2}$ Fraunhofer Institute for Reliability and Microintegration Berlin \\ ${ }^{3}$ University of Applied Sciences, Berlin
}

\section{Abstract}

Many silicon microsensors are using a thin silicon membrane with integrated piezoresistors as sensing element [1]. The performance of these micromechanical silicon sensors is strongly depending on the quality of the manufactured membranes such as geometrical parameters, roughness of membrane surface, uniformity of thickness etc..

Silicon membrane structures for sensors and actuators could be realized by using bulk micromachining technologies like $\mathrm{KOH}, \mathrm{TMAH}$ or DRIE (Deep reactive lon Etching). DRIE method is a well-known and very often used etching method in manufacturing of silicon sensors as it has many advantages such easy to use, no contact with etching solutions etc. [2]. The main disadvantages of this method are the non-uniformity of the etching rate over wafer, aspect ratio depending etching rate, tilting effect and notching [2].

Hence the signal quality of membrane-based MEMS sensors largely depends on his manufacturing process. Despite a maximum process control, it is not always possible to adjust the process parameters so that ideal manufacturing yield can be achieved. The dry etching does not deliver a fully homogenous result throughout the whole wafer. Caused by the inhomogeneity of the etching process different sensor signals are generated due to chip position on the wafer. In order to understand the influence of the inhomogeneity effect of dry etching on the sensor signal, the processed wafer has been fully measured using a profilometer and a mathematical model has been generated. Knowing the sensor position and the etching profile it is possible to reduce the distortion, predict the sensor signal and perform proper signal compensation.

\section{Introduction}

In MEMS one can find several structuring possibilities to fabricate e.g. membranes and beams. Beside wet it is dry etching here especially DRIE (Deep Reactive lon Etching), which can be used for the production of the membrane and beam structures. DRIE allows a broad variety control options for the process like process pressure, temperature, gas flows, plasma power, passivation and etch time [2]. All the parameters influence each other, so it is necessary to find compromises during production of the structures. One of these parameters is the homogeneity throughout the wafer.

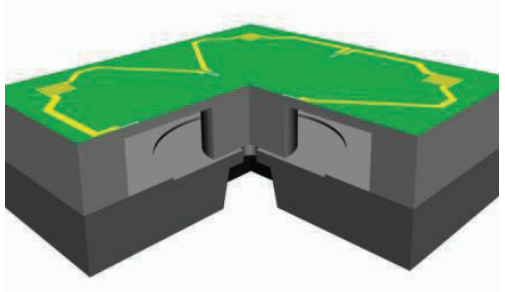

Figure 1: Sensor model with centerboss and dry etched sensor membrane using DRIE

Due to this inhomogeneity the chips have different membrane thickness from wafer edge to wafer centre. This effect can be even observed on a single chip. These chips not only show a different sensitivity compared to a centre-chip, but also can show undesired nonlinearities.

The sensor used in this study is a sensor developed for applications in harsh environments. The sensor concept, manufacturing and characterization have been published [3][4]. The used sensor consists of a DRIE etched sensor membrane with a centre-boss structure and four piezoresistors which are connected to a Wheatstone bridge. The membrane deflection and thereby change of resistivity of the piezoresistors is caused by a tip pushing on the centre of the membrane. Figure 1 shows a cross section of the used sensor [3][4]. 
For the mathematic calculation of the sensor signal it is necessary to know exactly the membrane thickness. Therefore the wafer (100-material, $100 \mathrm{~mm}$ diameter) was fully measured using a tactile profilometer. The data results in bowl shale shown in figure 2. This measurement was repeated for different process wafers and in figure 3 the membrane thickness was plotted dependent on the radius from the wafer centre. The red plot in this graph shows an approximation to these values. This plot can be described using a 3rd degree function $f(r)=A \cdot r^{3}+B \cdot r^{2}+C \cdot r+D$. The coefficients $A$, $B, C, D$ result after LMS (least mean square) procedure to $-4,30842 \mathrm{E}-13,-7,04557 \mathrm{E}-09$, $+0,000317875$ and $+49,76637057$ with an error of $2,2 \mu \mathrm{m}$. An approach with a higher degree (5th) improved the error by only $0.2 \%$. To further reduce the error one can assume that the wafer was not centred and therefore the measured values contain an offset in $\mathrm{x}$ and $\mathrm{y}$ direction. Extending the 3rd degree function with additional linear term to $f(r)=A r^{3}+B^{2}+C r+D+E x+F$ y leads to a reduced error by $\sim 20 \%$.

To find the coefficients the Generalized Reduced Gradient algorithm by Leon Lasdon and Allan Waren [5] was used. It is an iterative approximation to a defined optimum varying parameters, looking at the gradient of the derivatives, finding the influence of the parameter on the desired optimum.

With the found function it is possible to calculate the membrane thickness throughout the chip. This information can be used as input parameter for an FEM Simulation. The strain within the region of the piezoresistors can now be calculated much more precisely and therefore the whole signal of the Wheatstone bridge of the sensor.

\section{FEM Simulation}

To show how the different membrane thicknesses in the area of the piezoresistors influence the mechanical stress a FEM simulation was performed. The anisotropic parametric sensor model included three parameters, the minimum membrane thickness, the maximum membrane thickness and the angle of the chip to the centre of the wafer. With these three parameters it is not possible to describe the curvature of the sensor membrane, but as the curvature of the membrane for one

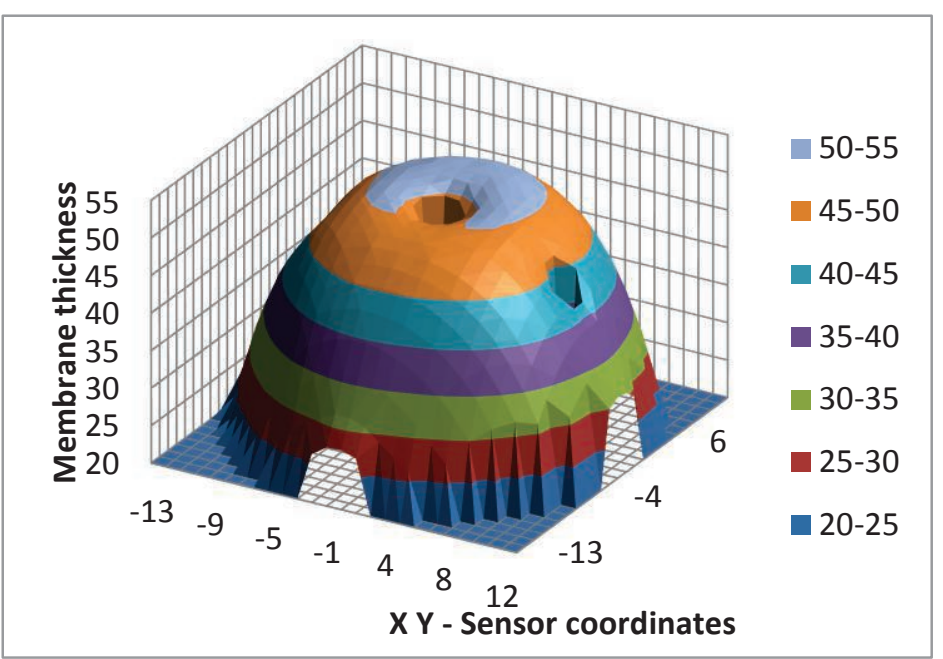

Figure 3 Membrane thickness throughout the wafer. The membrane thickness is calculated by substracting the etch depth from the initial wafer thickness. Caused by the mask design, there is no functional chip in the middle

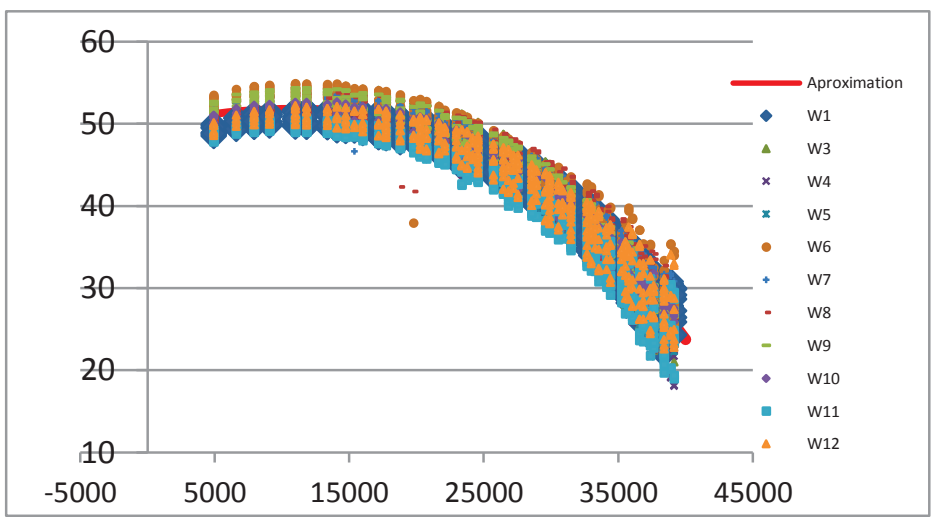

Figure 2: Measurement results of 12 DRIE etched wafer with the mathematical approximation of the membrane thickness across the wafer 
chip is very low it can be well approximated with a straight from maximum to minimum. The angle is needed for the anisotropic FEM model. In Figure 4 a cross section of the sensor model is shown. The sensor consists of a circular membrane with a centre boss. In the simulation a deflection on the topside of the centre boss was applied. The deflection causes a deformation of the silicon membrane and a stress concentration in the region of the piezoresistors. This stress is integrated over the area of the piezoresistors and this is used to calculate the sensor output and sensitivity. Figure 4 shows the sensor sensitivity for various membrane thicknesses (in $\mu \mathrm{m}$ ) depending on its deflection. It can be clearly seen that thicker membranes cause a higher sensor signal for the same deflection compared to thinner membranes.

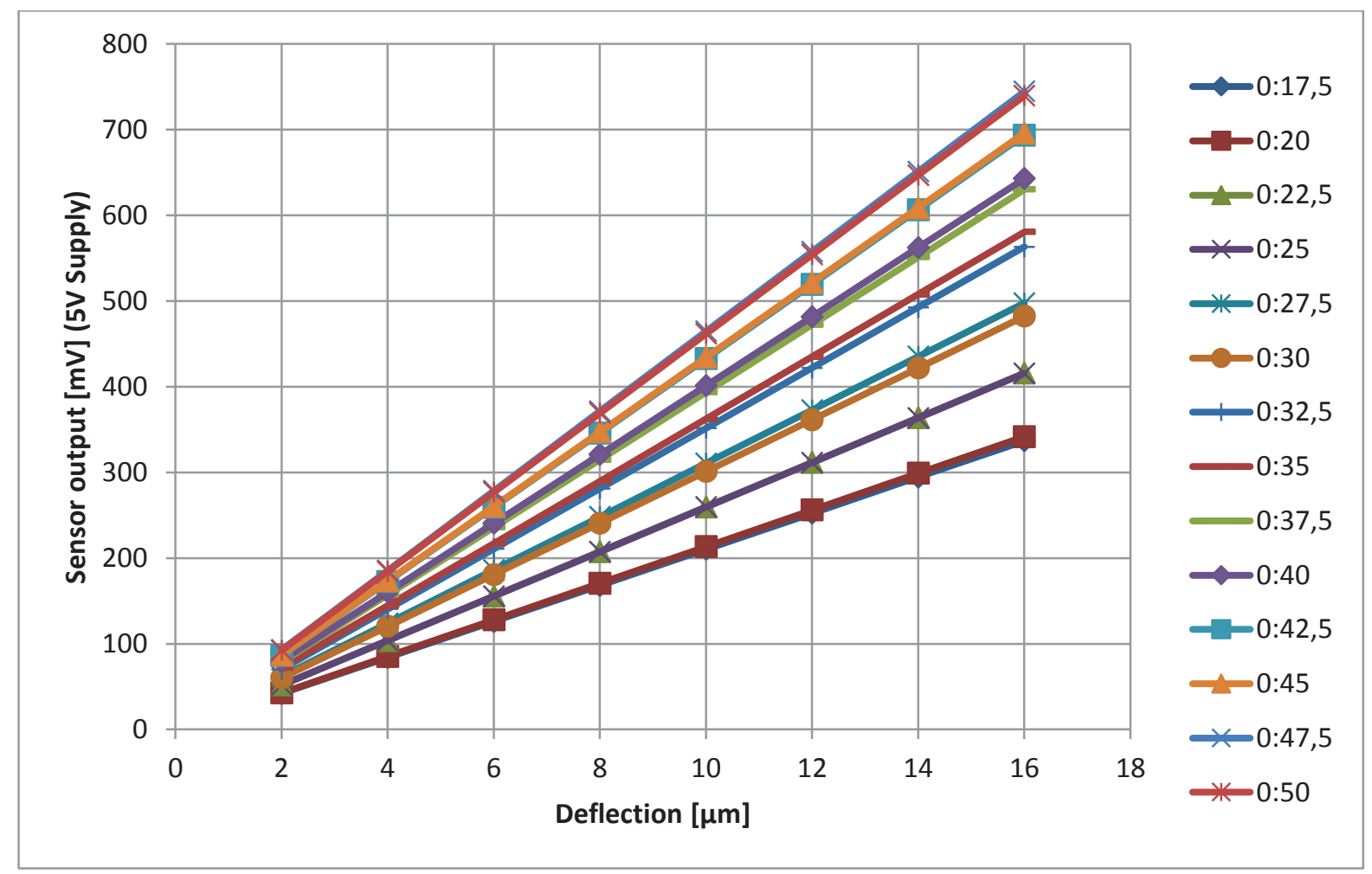

Figure 5 Simulation results for various membrane thicknesses (in $\mu \mathrm{m}$ ). The sensor outputs is for thick membranes and the same deflection are higher compared to the sensors with thinner membranes

But beside the etch non-uniformity DSE also causes rounded corners on the bottom of the membrane. A rounded membrane corner could reduce the mechanical stress there [6]. A simplified simulation model where we assure sharp edges will results in bad accordance between simulation and real measurement of the sensor. Therefor further models where generated describing the sensor membrane with rounded corners. Figure 6shows a comparison of a cross section of a real sensor with the simulation model.
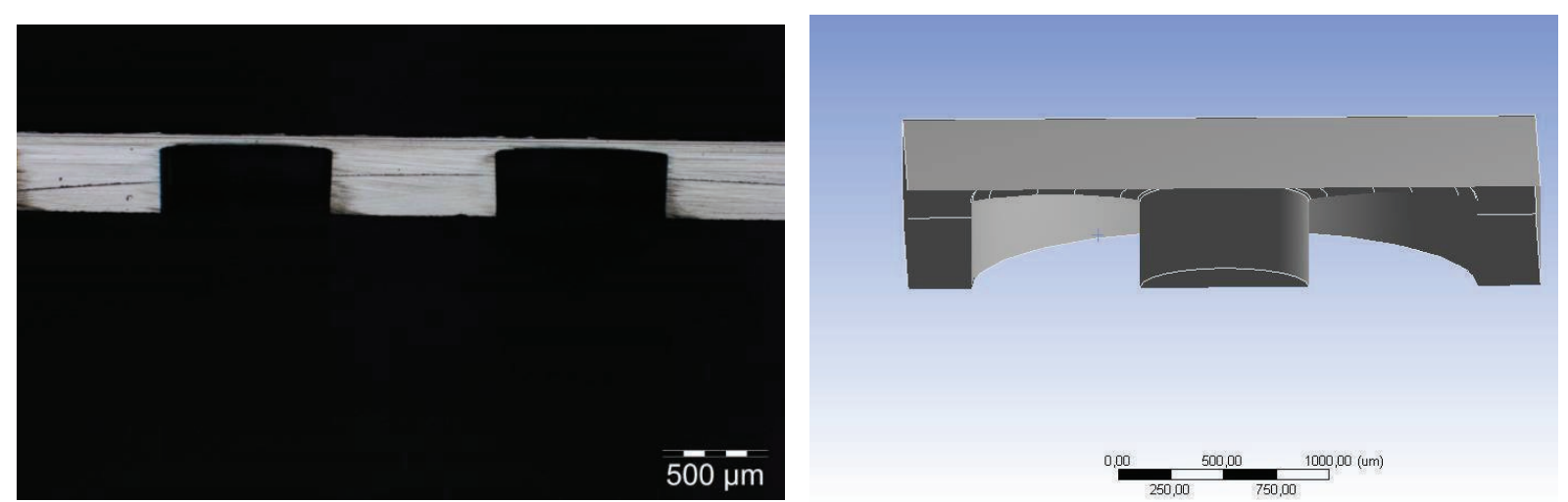

Figure 6: Comparison of rounded corners of the real sensor (left) and simulation model (right). The rounded corners will reduce the sensor output as the piezoresistors are located there 


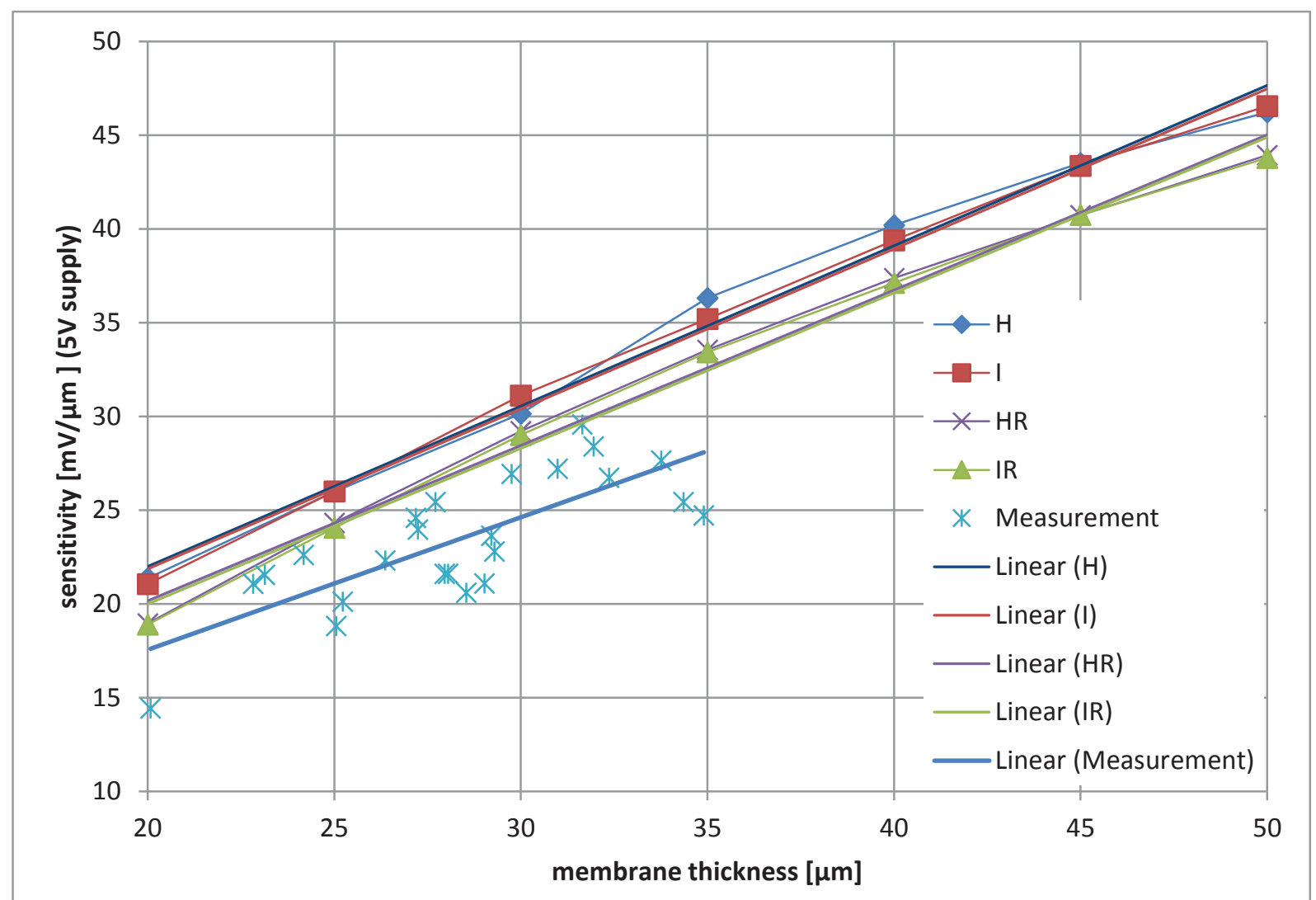

Figure 7: Comparison of sensor sensitivity for homogeneous (HR) and inhomogeneous (IR) sensor membranes with rounded membranes.

The simulation result show a strong sensitivity dependency of the membrane thickness. The inhomogeneous etched membrane with rounded corners show a lower sensitivity. The sensitivity loss because of inhomogeneous etched sensor membranes shows in comparison to a homogeneously etched membrane a difference up to $3 \%$.

\section{Measurement results}

The measurement was performed using a micro actuator with a needle that can be precisely moved in $z$ direction. The sensor is mounted in a ceramic socket and wire bonded to bond pads on the ceramic carrier which is electrically connected to measurements system. The measurement results of the measured sensor show a strong spread. Figure 8 shows the measured sensitivities of this sensor. Some chips show a very good and close accordance to the simulation.

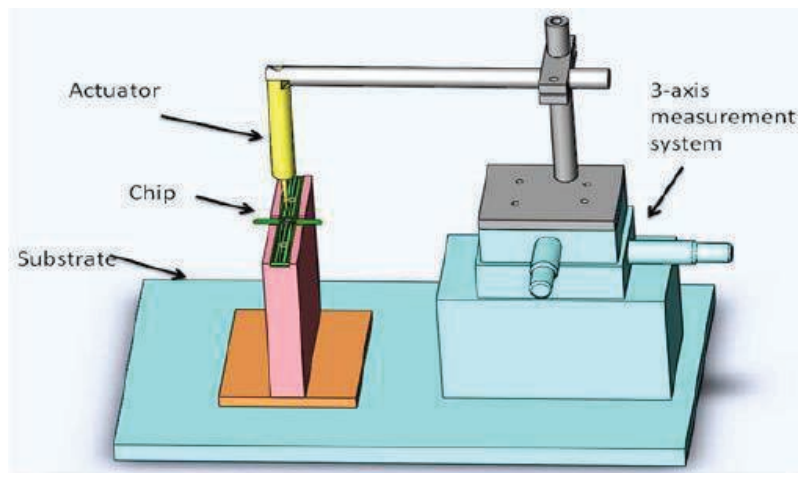

Figure 8: Actuator measurements setup with sensor chip. The actuator setup consists of 3-axis measurement system, actuator holder, actuator with control system, tested sensor holder

\section{Conclusion and Outlook}

With the theoretical and FEM sensor models considering the inhomogeneity in etching process it could be able to know exactly the performance of each sensor. This ability will help to improve the sensor compensation and to develop sensor with better performances. The simulation results prove that the inhomogeneity of the DRIE process even influences on a single chip. The measurement results match with the simulation results although the spread of the measurement is quite big. Here the measurement needs to be repeated and all the parameter checked. Especially the mounting of the sensor needs to be strengthen, the precision of the actuator and the membrane thickness need to be verified. 
The next step is to implement the mathematical model into a compensation system with the possibility to enter the chip ID and prove that the sensor output can be improved considering the process etch non-uniformity.

\section{References:}

[1] J. Fraden, "Handbook of modern sensors. Physics, design, and applications”. Springer, 2010

[2] S. J. Pearton, " Handbook of advanced plasma processing techniques". Springer, 2000

[3] H.-D. Ngo, B. Mukhopadhyay "Liquid-Free, Piezoresistive, SOl-Based Pressure Sensor for High Temperature Measurements up to $400{ }^{\circ} \mathrm{C}^{\prime}$, IEEE Sensors, Taiwan, 2012

[4] H.-D. Ngo, A. Giuliania, "SOl-based, High Reliable Pressure Sensor with Floating Concept for High Temperature Applications" Eurosensors, Italy, 2014

[5] L. Lasdon, A.Waren Design and Testing of a Generalized Reduced Gradient Code for Nonlinear Programming, Journal ACM Transactions on Mathematical Software (TOMS), 1978

[6] H.-D. Ngo, A.-T. Tham, M. Simon, E. Obermeier, "Corner rounding to strengthen silicon pressure sensors by using DRIE". IEEE Sensors Conference, Lecce, Italy, 2008 